\title{
Energy Optimal Control to Approach Traffic Lights
}

\author{
Andreas Lawitzky, Dirk Wollherr, and Martin Buss
}

\begin{abstract}
In this paper energy optimal solutions for the approach of red traffic lights are derived. As cars waste most of the fuel in city traffic and especially in queuing at traffic lights, the presented framework provides solutions to save fuel and to protect the environment. The solutions are obtained using the definition of spent physical work which has to be minimized. It covers both cases, that the time of switching of the traffic lights is known and that the time of switching can only be modeled as a stochastic process. For a known time of switching a continuous solution is derived using Pontryagin Minimum Principle; in the stochastic case a modified Bellmann equation is formulated. The latter is solved with dynamic programming techniques. The presented solutions can be used for autonomous driving as well as for driving assistant systems. Simulation results show the potential savings using the presented approach.
\end{abstract}

\section{INTRODUCTION}

Future transportation systems need to increase fuel efficiency as cheap energy resources are getting rare. But not only economics is a convincing reason to increase fuel efficiency. Every investment in fuel economy pays off twice, in decreased costs for the driver and in the protection of the environment. To fulfill the requirement of higher efficiency many research technologies have been developed in recent years to decrease fuel consumption of vehicles. The range of optimizations include more efficient engines, adjusted vehicle designs and lighter chassis. This way the losses e.g. due to friction and air drag are aimed to be reduced. Furthermore researchers put effort in developing intelligent traffic management systems of cities.

Either way, the driving style plays its role in energy efficiency as well. Economic driving has been shown to reduce fuel consumption significantly [1], hence saving money and protecting the environment. Therefore newer cars come with several driving assistance technologies that help the driver to minimize energy demand. As an example many automobile companies include a gear shift indicator (GSI) system that suggests the most efficient gear. The start-stop-systems which can be found in recent cars can also be seen as such a system.

On the other side, research has been made regarding the inter-connection of vehicles and the infrastructure. They aim to make cities smart by means of maximizing throughput while minimizing fuel consumption e.g. by coordinated switching of traffic lights. The next big step in this domain, vehicular ad-hoc networks systems like Car2X or infrastructure-to-vehicle (I2V) have not yet found their way in production vehicles. Up to now, these systems are limited to research areas as challenges like reliable communication and security of the system persist.

The authors are with the Institute of Automatic Control Engineering (LSR). Dirk Wollherr is also with Institute for Advanced Study (IAS). Both institutes are with the Technische Universität München, D-80290 München, Germany. $\{a . l a w i t z k y, d w$, mb\}etum.de
Apart from conventional vehicles, the domain of intelligent transport systems received much attention in recent years, by addressing the problem of completely autonomous driving. The motivation behind these autonomous systems is that they can continuously collect and process large amounts of data to increase safety, comfort and fuel efficiency, e.g. by incorporating congestion and traffic lights information directly into motion planning. A notable step in autonomous driving is the famous DARPA Urban challenge [2] - an autonomous driving challenge. While the main idea at this point was to handle all traffic situations correctly, research goes now one step further into optimization of driving behavior.

This paper concentrates on energy optimal approaching red traffic lights. This is done by providing solutions to the optimal control problems. It covers situations where $\mathrm{I} 2 \mathrm{~V}$ is present and the switching time is known exactly as well as to situations where the switching time is only known with arbitrary uncertainty. An example implementation of this framework that provides optimal solutions for the approach of traffic lights is presented and validated by simulations.

Note that, although the content described herein is about cars, it is perfectly applicable to other traffic participants as bicycles or even trains.

\section{RELATED WORK}

The challenge of energy efficient transportation systems has been discussed from different perspectives. Recently, many researchers e.g. [3] have focused on optimal switching of traffic lights based on historic traffic data or on current traffic monitoring. These systems aim to minimize the interruption for intersecting traffic flows caused by traffic lights.

The problem how to optimize the approach of traffic lights has been addressed as well. In [1] speed profiles have been compared to their energy demand. The work investigated the effect of energy aware driving with driving assistance systems. However, only a limited set of profiles has been considered, so it lacks mathematical justification that the presented speed profile is optimal.

Another system to control the approach of traffic lights is presented in [4]. Without discussing the optimal control problem behind it, the authors describe a solution using constant negative acceleration. This solution is suboptimal as will be shown later. In [5] a similar approach is introduced, that assists the driver with an optimal speed to reach the traffic lights exactly when they are switching, what helps to save fuel. Unfortunately, the approach does not consider dynamic constraints, thus allowing jumps in velocity (i.e. infinitely high accelerations) which is not applicable in real world scenarios. The influence of the correct gear choice on fuel consumption at an traffic lights approach has been discussed in [6], again using a set of speed profiles. 
In [7] the problem of crossing multiple traffic lights with minimal energy is discussed. The authors aim to find a single, constant speed to get through a series of traffic lights without having to stop using a predictive cruise control algorithm. However, as a solution of this problem is often infeasible a model-predictive-control architecture is used.

Other research has been done in predicting the switching of traffic lights without communication to the infrastructure. With the help of a camera of a mobile phone the SignalGuru [8] makes assumptions about the future state of the traffic lights. Communication between multiple vehicles equipped with these mobile phones allowed to obtain high precision. Another approach is to learn the phase of traffic light schedules from speed profiles of multiple vehicles as in [9].

A communication framework to $\mathrm{I} 2 \mathrm{~V}$ is set up in [10]. Furthermore, a possible human-machine-interface (HMI) to assist the driver is described. In [11] the concept of a traffic light assistant is discussed by means of acceptance of the driver using a simulator survey.

In contrast to the former approaches, [12] proposes a concept of energy optimal trajectories for trains. The communication issues and a possible framework is discussed to send optimal trajectories to the drivers of trains.

The common approach to energy optimal trajectories is to use an linear-quadratic-regulator (LQR) system [13]. With its nice mathematical properties, LQR systems can be solved completely. Either way, the obtained results do not comply with the definition of physical work and kinetic energy.

More recently, a similar problem as discussed here can be found in [14]. The authors tackle the problem with an optimal control approach minimizing a cost function weighting time spent, high accelerations and idling using deterministic dynamic programming. Similar to this work, the authors give an optimal control trajectory even for the case that only incomplete knowledge about signal phase times is available. The results and the approach however differ as their cost function is only heuristically motivated.

So none of the former works derived a solution to the optimal control problem based on the definitions of physical work. The solution with known time of switching of the traffic lights is shown for completeness, although being quite straight forward with Pontryagin's minimum principle. But, this work also provides solutions to the non-trivial optimal control problem that the knowledge of the time of switching can only be modeled with a probability distribution.

\section{PROBLEM STATEMENT}

The focus of this paper is to find the energy optimal control input to approach red traffic lights. Assuming a frictionless driving model the presented framework solves an optimization problem with restrictions in input and state space. It aims to minimize the physical work that has been spent while the lights remained red plus the work that needs to be spent to accelerate back to the desired speed.

Unlike former works where for the sake of ease squared energy functions are used, it is strictly based on the definitions of kinetic energy. It respects the fact that only acceleration is costly not braking (which is still true for most of today's cars). However, note that the presented approach can easily be extended to friction-aware models and vehicles with (partially) regenerative braking. For this approach only a single vehicle is considered, since interaction is not in the scope of this work. It is also assumed that only a single red phase of the traffic lights has to be regarded.

In the following, the one-dimensional system is modeled with $x_{1}$ being the position of the bumper of the vehicle, and $x_{2}$ being the speed of the regarded vehicle while $u$ is the input acceleration. Both state variables are packed into a state vector $\boldsymbol{x}$ which fulfills the equations of a double-integrator system

$$
\begin{aligned}
\boldsymbol{x} & =\left[\begin{array}{ll}
x_{1} & x_{2}
\end{array}\right]^{\top} \\
\dot{\boldsymbol{x}} & =f(\boldsymbol{x}, u)=\left[\begin{array}{ll}
x_{2} & u
\end{array}\right]^{\top} .
\end{aligned}
$$

The optimal control problem needs to respect the following constraints of the state space $\mathcal{X}=\left[\begin{array}{ll}\mathcal{X}_{1} & \mathcal{X}_{2}\end{array}\right]^{\top}$ and the input space $\mathcal{U}$

$$
\begin{aligned}
x_{2} & \in \mathcal{X}_{2}=\left[0, v_{\text {Max }}\right] \\
u & \in \mathcal{U}=\left[u_{\text {Min }}, u_{\text {Max }}\right]
\end{aligned}
$$

where $v_{\mathrm{Max}}>0$ and $u_{\mathrm{Min}}<0<u_{\mathrm{Max}}$.

The red traffic lights are detected at $t=0$. The initial condition is denoted as

$$
\begin{aligned}
& x_{1}(0)=0 \\
& x_{2}(0)=v_{0} \in \mathcal{X}_{2}
\end{aligned}
$$

as illustrated in Fig. 1. The distance between $x_{1}=0$ and the stop line is $d$.

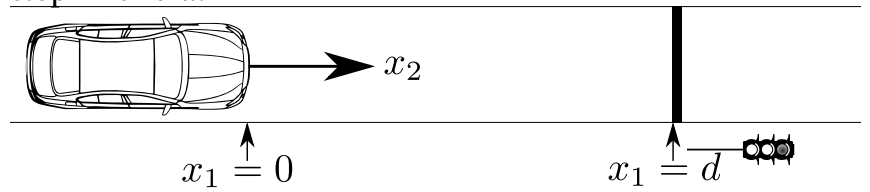

Fig. 1. At $t=0$ the vehicle detects the red traffic lights. Its initial state is denoted $\left[0, v_{0}\right]^{\top}$. The distance to the traffic lights is $d$.

The optimization aims to minimize the physical work that has to be applied in this situation, respecting the constraints.

The time the traffic lights switches from red to green is denoted as $T>0$. So, the work that has to be spent for a certain trajectory can be separated into the work that has been used in the interval $[0, T]$, denoted as $W_{\text {Accelerate, }}$ and the work that has to be supplied after $t=T$, denoted as $W_{\text {Remaining, }}$, to reach a desired speed. Hence, the optimization problem is to minimize

$$
W_{\text {Accelerate }}+W_{\text {Remaining }} \rightarrow \min .
$$

The remainder derives the solution to this problem for both, that $T$ is known and that $T$ is unknown.

\section{APPROACH}

Using the definition of the physical work $W$, make a separation of acceleration work and braking work

$$
\underbrace{\int_{0}^{T} m u(t) x_{2}(t) \mathrm{d} t}_{W}=\underbrace{\int_{0}^{T} m u^{+}(t) x_{2}(t) \mathrm{d} t}_{W_{\text {Accelerate }}}+\underbrace{\int_{0}^{T} m u^{-}(t) x_{2}(t) \mathrm{d} t}_{W_{\text {Brake }}}
$$


with $m$ being the mass of the vehicle and

$$
\begin{aligned}
& u^{+}(t)=u(t) \Theta(u(t)) \\
& u^{-}(t)=u(t) \Theta(-u(t)),
\end{aligned}
$$

where $\Theta$ is the unit step function. Denote the initial kinetic energy as $E_{0}$. The energy at the switching time $T$ is written as $E_{T}$ and defined using the energy equilibrium as

$$
E_{T}-E_{0}=W_{\text {Accelerate }}+W_{\text {Brake }}
$$

Without loss of generality, it is assumed that the desired energy of the vehicle is again $E_{0}$, so

$$
W_{\text {Remaining }}=E_{0}-E_{T} \text {. }
$$

With (6) and (7), the problem (5) can be similarly rewritten as

$$
-W_{\text {Brake }}=-m \int_{0}^{T} u^{-}(t) x_{2}(t) \mathrm{d} t \rightarrow \min
$$

For the easy of notation let $m=1$ without losing generality.

Of course, it is assumed that it is still possible to brake enough by the time the traffic lights are detected. Hence, the stop line has not been crossed while the lights are still red

$$
t=\min \left\{\frac{v_{0}}{u_{\mathrm{Min}}}, T\right\}: \quad \frac{1}{2} u_{\mathrm{Min}} t^{2}+v_{0} t \leq d .
$$

In the remainder, the optimization problem (8) is solved, first for the case that the time of switching is known. Afterwards, it is solved for the case that the knowledge about $T$ can only be stochastically modeled.

\section{A. Optimal Control with Known Switching Time}

In this section the optimal control problem is solved under the assumption that the switching time $T$ is known. The end condition is set to

$$
\begin{aligned}
& x_{1}(T)=d \\
& x_{2}(T) \text { free, }
\end{aligned}
$$

where the solution is later extended for a free end position $x_{1}(T) \leq d$. Note, that the case that the detected red lights are far enough away that the vehicle does not have to react is trivial and hence not considered here, so assume

$$
T v_{0}>d \text {. }
$$

1) Solving the Optimization Problem: With (8) the cost function is consequently defined as

$$
J=-\int_{0}^{T} u^{-}(t) x_{2}(t) \mathrm{d} t .
$$

As shown later, the state constraint (2) is never active under (9) and is hence not considered in the calculation.

The corresponding Hamiltonian [15] of (12) is with (1)

$$
H=-u^{-} x_{2}+\lambda_{1} x_{2}+\lambda_{2} u,
$$

omitting time dependencies. Evaluating the costates $\boldsymbol{\lambda}=$ $\left[\begin{array}{ll}\lambda_{1} & \lambda_{2}\end{array}\right]^{\top}$

$$
\begin{aligned}
& \dot{\lambda}=-\nabla_{\boldsymbol{x}} H=-\left[\begin{array}{ll}
0 & -u \Theta(-u)+\lambda_{1}
\end{array}\right]^{\top} \\
& \dot{\lambda}_{1}=0 \Rightarrow \lambda_{1}=c \Rightarrow \dot{\lambda}_{2}=u \Theta(-u)-c
\end{aligned}
$$

is concluded, with an unknown constant $c$.

Using Pontryagin's minimum principle (PMP) [15], $H$ is minimized w.r.t. $u$. It is obtained that the input $u$ is

$$
u(t)= \begin{cases}u_{\mathrm{Max}}, & \lambda_{2}<0 \\ 0, & 0 \leq \lambda_{2} \leq x_{2} \\ u_{\mathrm{Min}}, & \lambda_{2}>x_{2} .\end{cases}
$$

From the transversality condition with (10) the final costate is $\lambda_{2}(T)=0$. With this, it is concluded that in an area around $T$ the input $u \geq 0$, so the derivative of the second costate is $\dot{\lambda}_{2}=-c$.

Respecting the start conditions (4) and restrictions (2) and (11) only $c>0$ is allowed. Therefore, it is necessary that there is an unknown input switching time

$$
\exists t_{\mathrm{sw}} \in(0, T): \quad \lambda_{2}\left(t_{\mathrm{sw}}\right)=x_{2}\left(t_{\mathrm{sw}}\right) .
$$

Hence, it is concluded with (4) that the optimal strategy is to perform a braking till $t=t_{\mathrm{sw}}$ with maximum deceleration and to keep the speed afterwards

$$
x_{2}(t)= \begin{cases}u_{\mathrm{Min}} t+v_{0}, & t \leq t_{\mathrm{sw}} \\ u_{\mathrm{Min}} t_{\mathrm{sw}}+v_{0}, & t>t_{\mathrm{sw}}\end{cases}
$$
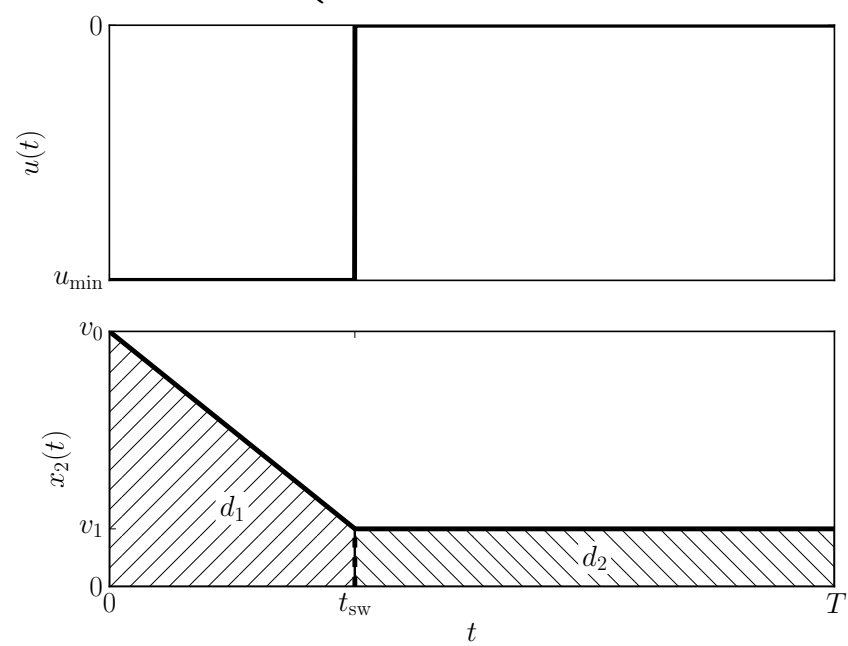

Fig. 2. The energy optimal control is to perform a braking with $u_{\mathrm{Min}}$ till $t_{\mathrm{Sw}}$ and keep the speed afterwards. $t_{\mathrm{Sw}}$ can be calculated easily using the area under the speed graph, defining $d_{1} \square$ and $d_{2} \square$.

2) Finding Input Switching Time $t_{\mathrm{sw}}$ : A geometric approach can be used to find $t_{\mathrm{sw}}$ as in Fig. 2. With

$$
\begin{aligned}
d & =d_{1}+d_{2} \text { where } \\
d_{1} & =v_{0} t_{\mathrm{sw}}+\frac{1}{2} u_{\mathrm{Min}} t_{\mathrm{sw}}^{2} \\
d_{2} & =\left(v_{0}+u_{\mathrm{Min}} t_{\mathrm{sw}}\right)\left(T-t_{\mathrm{sw}}\right)
\end{aligned}
$$

the input switching time $t_{\mathrm{sw}}$ can be obtained as

$$
t_{\mathrm{sw}}=T-\sqrt{T^{2}+\frac{2}{u_{\mathrm{Min}}}\left(v_{0} T-d\right)} .
$$

Hence the minimal dissipated energy is

$$
W_{\text {Brake }}=\frac{1}{2}\left(v_{0}^{2}-\left(u_{\mathrm{Min}} t_{\mathrm{sw}}+v_{0}\right)^{2}\right) .
$$

Note that restriction (2) is never active given (9). Therefore, it was not considered in the Hamiltonian (13). 
3) Free endpoint $x_{1}(T)$ : Eq. (16) can be generalized with its end state using the general free end position $x_{1}(T)$ according to

$$
t_{\mathrm{sw}}=T-\sqrt{T^{2}+\frac{2}{u_{\mathrm{Min}}}\left(v_{0} T-x_{1}(T)\right)} .
$$

It is obvious that for reasons of monotonicity $t_{\mathrm{sw}}$ in (18) is minimal for maximal $x_{1}(T)$. $W_{\text {Brake }}$ is minimal for $x_{1}(T)=$ $d$ like assumed in IV-A.

With this, the optimal control trajectory is obtained for the approach of traffic lights when $T$ is known. While this solution was given for completeness, the related problem how to behave optimal with non-exact knowledge about the time of switching of the traffic lights $T$ is shown in the remainder.

\section{B. Optimal Control for Stochastically Modeled Knowledge}

This section now discusses the optimal control problem (8) in a scenario that the time of switching of the traffic lights $T$ is not perfectly known. However, it is assumed that the knowledge about $T$ can be modeled using a stochastic distribution. These are characterized by a probability density function $\rho(T)$.

This scenario is extremely important if $\mathrm{I} 2 \mathrm{~V}$ systems are not available. So, this set-up is facing reality that hardly any of today's traffic lights are equipped with $\mathrm{I} 2 \mathrm{~V}$ devices.

In the remainder, it is assumed that there exists a finite

$$
T_{\text {Max }}=\sup \{T \mid \rho(T) \neq 0\},
$$

so that $T$ is bounded. Loosely speaking, it is known that the lights will switch before $T_{\mathrm{Max}}$. Of course, the constraints (9) and (10) have to be adjusted appropriately. With this the energy optimal trajectory is searched.

Note, that this case is special as accelerating does not add anything to the costs as in (12). Put differently, it is not of relevance how the acceleration trajectory after the switching of the traffic lights looks like. Consequently it is obvious that we are only interested in the trajectory if the traffic lights do not switch in $\left[0, T_{\mathrm{Max}}\right)$. As $T$ is the actual (but unknown) switching time, it is optimal to behave according to the available knowledge at this time step for $t<T$ and then, after $T$ to re-accelerate. The following finds the solution to the optimal control problem according to the available knowledge at each time step.

1) Reformulation of the Optimal Control Problem: In the remainder the time is discretized in $n$ steps, each of duration $\Delta t$. So, the modified optimization problem can loosely be described with: What is the best input to drive for the next timespan $\Delta t$ with the currently available knowledge about $T$ ? It is assumed that the vehicle can only react on green lights after a time step, not in between.

The chance that traffic lights will not switch between the current time step $k$ and the following time step $k+1$ under the condition that they have not switched up to $k$ is denoted as $\beta(k)$ and defined as

$$
\beta(k)=1-\frac{\int_{k \Delta t}^{(k+1) \Delta t} \rho(T) \mathrm{d} T}{\int_{k \Delta t}^{\infty} \rho(T) \mathrm{d} T}=1-\frac{\int_{k \Delta t}^{(k+1) \Delta t} \rho(T) \mathrm{d} T}{\int_{k \Delta t}^{T_{\mathrm{Max}}} \rho(T) \mathrm{d} T} .
$$

This probability that the light will not switch in the interval $[k \Delta t,(k+1) \Delta t]$ uses the fact, that a probability density function is cropped and scaled if the event did not happen up to point in time. The detailed proof of this can be found in the appendix of this paper.

So the expected cost consists of the actual cost for the action $\phi\left(\boldsymbol{x}, u_{k}\right)$ and the expected cost of the situation of the next time step. This leads to a modified Bellmann equation using the system definition (1) for $k=0 \ldots(n-1)$

$$
\begin{gathered}
J_{k}^{*}\left(\boldsymbol{x}_{k}\right)=\min _{u_{k}}\left[\phi\left(\boldsymbol{x}_{k}, u_{k}\right)+\beta(k) J_{k+1}^{*}\left(f\left(\boldsymbol{x}_{k}, u_{k}\right)\right)\right] \\
\text { with } J_{n}^{*}\left(\boldsymbol{x}_{n}\right)=J_{n}^{*}=0 .
\end{gathered}
$$

Note that $\beta(k)$ is a function of the discretized time $k$. In each time step, this function incorporates the knowledge that the traffic lights did not switch yet, but minimizes the cost for the expectation.

2) Implementation of the Solver: In order to find the energy optimal input trajectory a grid based solution is used in the presented framework. This type of strategy is also commonly known as discrete, stochastic dynamic programming and described e.g. in [13].

An illustration of such a grid can be seen in Fig. 3. The

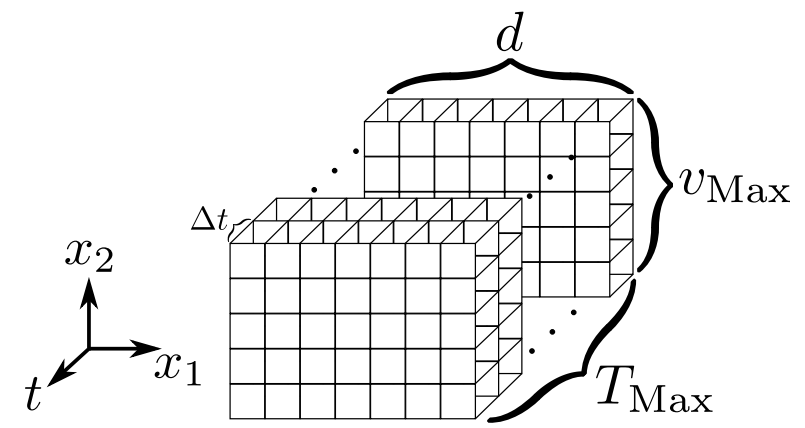

Fig. 3. A grid is representing the three dimensions of time and state space. Each cell stands for a discrete point in this coordinate system. The size of the grid is given by maximum distance to the traffic lights, the maximum speed and the upper bound for the switching time.

grid consist of three dimensions, representing the time in the interval $\left[0, T_{\mathrm{Max}}\right]$ and the two-dimensional state space $\boldsymbol{x}$, in $[0, d]$ and $\left[0, v_{\mathrm{Max}}\right]$ respectively as restrictions apply analogous to the above. Each cell in this grid represents a point in time $k$ with the state $\boldsymbol{x}_{k}$. Assuming small $\Delta t$ the input $u_{k}(t)$ can be regarded as constant $u_{k}(t)=u_{k}$. This decreases the computational demand to find the optimal solution. For a piecewise constant input $u_{k}$ the cost for the action simplifies to $\phi\left(\boldsymbol{x}_{k}, u_{k}\right)=u_{k} \Delta t\left(x_{2, k}+\frac{1}{2} u_{k} \Delta t\right)$.

With this framework arbitrary probability density functions can be considered, if they fulfill the condition in (19). As examples the optimal control trajectories in case of uniform probability distributions and in case of a triangular distribution are presented.

3) Uniform Probability Distribution: In this section the special case is discussed, that $\rho$ is distributed uniformly, as shown in the top plot of Fig. 4. Note that this case occurs especially if the length of the red light phase $T_{\mathrm{Max}}$ is known (or can be overestimated), but the time the vehicle first 
detects the lights in respect to the phase is unknown. So the uniform distribution is of great practical use.

For $\rho$ being uniform, the equation (20) simplifies to

$$
\beta(k)=1-\frac{\Delta t}{T_{\mathrm{Max}}-k \Delta t} .
$$

The obtained resulting optimal input and the corresponding state trajectories are sketched in Fig. 4.

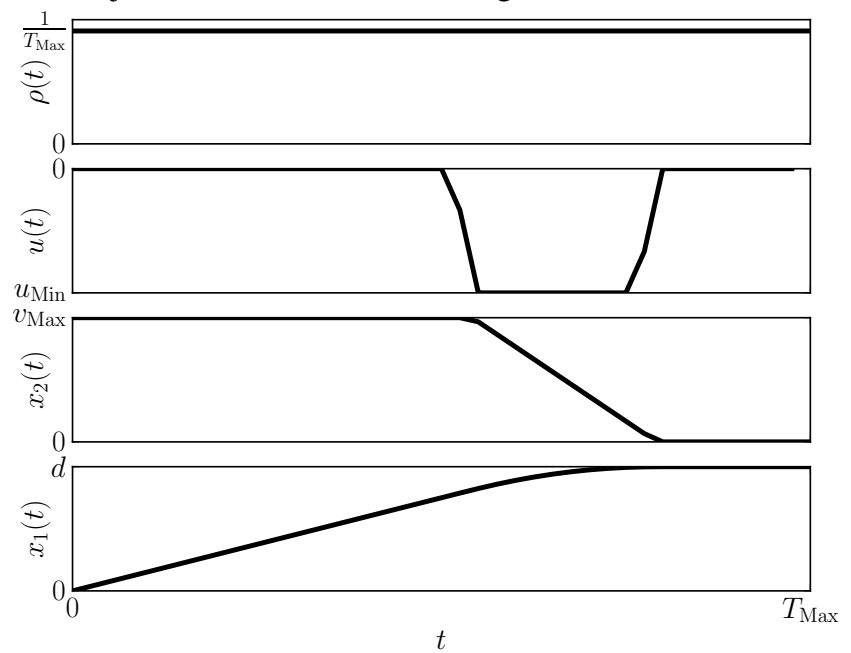

Fig. 4. In case of a uniform distribution as sketched in the top plot, the obtained optimal control trajectories are as shown in the lower plot. The second plot presents the trajectory of the optimal input, the two lower plots the resulting trajectories of the state.

4) Triangular Probability Distribution: As an academic example, a triangular distributed probability density function like in the top plot of Fig. 5 is regarded in this section. Again, the optimal input and state trajectories are shown in Fig. 5.

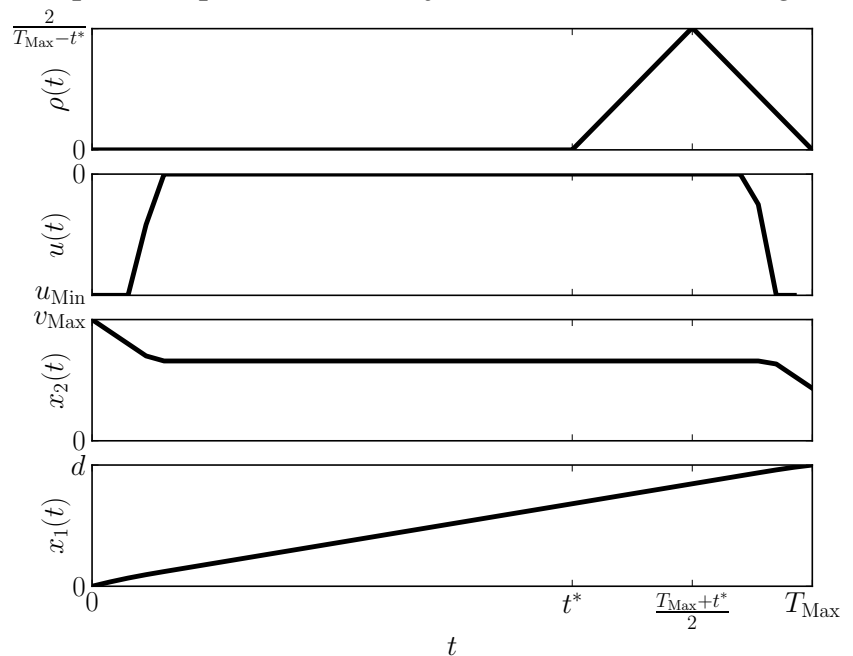

Fig. 5. Optimal control trajectory in case of triangular $\rho$ as shown in top plot. The lower plots show the optimal input $u$ and the optimal two state trajectories $x_{1}$ and $x_{2}$ respectively.

The last section gave a qualitative overview over the optimal solutions of the approach of red traffic lights. In the following sections the potential fuel savings of the optimal trajectories are discussed.

\section{RESULTS}

The presented algorithm to energy optimal approach to red traffic lights has been exemplary implemented. As discussed in IV-B.2 the solution is realized as a three-dimensional grid.
For these scenarios the parameters of this grid have been chosen as follows. It is assumed that $m=1000 \mathrm{~kg}, T_{\mathrm{Max}}=$ $20 \mathrm{~s}$, the maximum distance to the line is $x_{1, \max }=200 \mathrm{~m}$ and the maximum speed is $x_{2, \max }=15 \mathrm{~m} / \mathrm{s}$. The input space is $\mathcal{U}=\left[u_{\mathrm{Min}}, u_{\mathrm{Max}}\right]$ where $u_{\mathrm{Max}}=-u_{\mathrm{Min}}=3.0 \mathrm{~m} / \mathrm{s}^{2}$.

For this grid, the input space has been discretized in 13 steps, so $\Delta u=0.25 \mathrm{~m} / \mathrm{s}^{2}$. Furthermore the time space is represented by 41 discrete time steps, resulting in $\Delta t=0.5 \mathrm{~s}$.

From the above parameters the discretization of the state space is implied. With this, the speed is consequently sampled in 121 steps according to $\Delta x_{2}=\Delta u \Delta t=0.125 \mathrm{~m} / \mathrm{s}$. Likewise the position $x_{1}$ has to be discretized with $\Delta x_{1}=$ $\frac{1}{2} \Delta u \Delta t^{2}=0.03125 \mathrm{~m}$ resulting in 6401 steps.

The code has been tested on a standard PC with an Intel i5 with $3.30 \mathrm{GHz}$ on Linux. With the presented, single-threaded exemplary implementation in Python it takes $37 \mathrm{~s}$ to obtain the whole grid with the above dimensions. However with a multi-threaded implementation in a compiled language online capability is expected even for finer grids.

With this implementation optimal trajectories can be obtained which are in the remainder compared to other possible trajectories. In Fig. 6 the obtained optimal input trajectory is compared to a constant acceleration trajectory as well as to an optimal trajectory for known $T=T_{\text {Max }}$, as described in Sec. IV-A. The plot in Fig. 6a shows an histogram of the $10^{7}$ samples illustrated in bins that have been used, and below the compared input trajectories with the corresponding course of energy. As reported in Tab. $6 \mathrm{~b}$ the optimal trajectory outperforms the other exemplary functions by more than $25 \%$. The optimal trajectory for a triangular probability distribution function is compared in Fig. 7 to the other functions. Again, the optimal trajectory significantly outperforms the other functions as listed in Tab. 7b.

Note that the presented probability distributions are just for illustration purposes. Arbitrary probability distributions that model the perception and prediction uncertainties can be used in this framework.

\section{CONCLUSION AND FUTURE WORK}

This paper presented a framework to approach red traffic lights optimally w.r.t. energy consumption. The optimal control problem is deduced from the definition of physical work. It derives optimal trajectories for the case the time of switching of the lights is known as well as for the case that the switching can only be stochastically modeled. It has been shown that there are notable potential energy savings in the optimization. The system has been created for both, as a future driving assistant system and as part of an intelligent, autonomous vehicle.

Either way, the proposed approach is yet limited to simulation results. Hence, future work includes implementation in real life environments. With a more more sophisticated implementation on-line capability is expected for arbitrary probability density functions.

\section{APPENDIX}

The following definitions are used to show that a probability density function is only cropped and scaled if the 

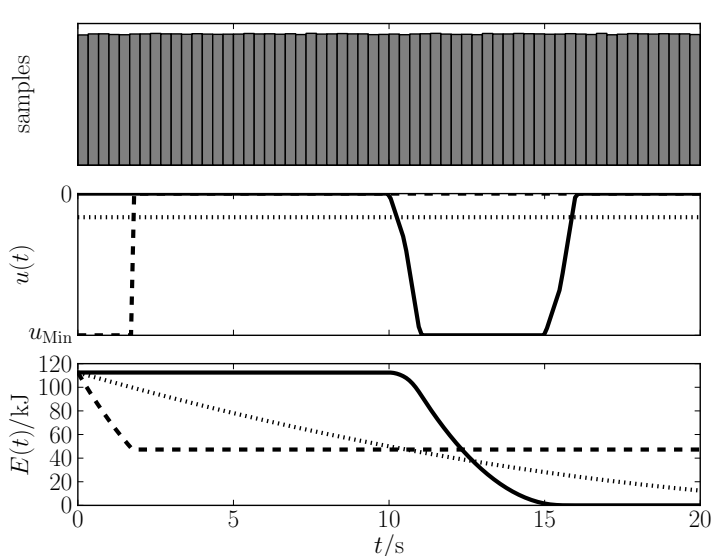

(a) In this plot an histogram of the sampled probability distribution is shown. Below, the optimal input (solid), a constant acceleration solution (dotted) and the optimal solution for the worst case $T=T_{\mathrm{Max}}$ (dashed) are sketched. The plots show the acceleration and the energy trajectory over time.

\begin{tabular}{lccc}
\hline Trajectory & optimal & CA & $\begin{array}{c}\text { opt. for } \\
T=T_{\text {Max }}\end{array}$ \\
\hline avg. energy loss/kJ & 43.7 & 58.3 & 62.6 \\
\hline avg. saving & - & $25.2 \%$ & $30.2 \%$ \\
\hline
\end{tabular}

(b) The optimal solution for the uniform probability density function outperforms the other candidates taking the average over all samples.

Fig. 6. Comparing the optimal solution for a uniform probability distribution to a constant negative acceleration (CA) trajectory and to the optimal trajectory for the case $T=T_{\mathrm{Max}}$. Although other solutions have less energy loss at some samples, the optimal solution outperforms them in average. event has not occurred up to a point $\mu \in \mathbb{R}$. The probability density function $\rho(T)$ is given and the random variable is $X$. Furthermore, let $a, b \in \mathbb{R}$ and the interval $I=[a, b]$.

With the definition of the conditional probability

$$
\begin{aligned}
P(X \in I \mid X>\mu)= & \frac{P(X \in I, X>\mu)}{P(X>\mu)} \\
= & \begin{cases}\frac{P(X \in I)}{P(X>\mu)} & \mu \leq a \\
\frac{\int_{\mu}^{b} f(t) \mathrm{d} t}{P(X>\mu)} & a<\mu<b \\
0 & b \leq \mu .\end{cases}
\end{aligned}
$$

Note that with $\alpha=P(X>\mu)^{-1}$

$$
\frac{P(X \in I)}{P(X>\mu)}=\alpha P(X \in I) \text {. }
$$

So, in particular the probability density function $\rho(T)$ is cropped at $\mu$ and $\rho(T)$ is scaled by factor $\alpha$ for $T>\mu$.

\section{ACKNOWLEDGMENT}

The authors gratefully acknowledge financial support of this work by the BMW Group, Munich, Germany. This work is supported in part by the DFG excellence initiative research cluster Cognition for Technical Systems - CoTeSys and by the Institute for Advanced Study (IAS), Technische Universität München.

\section{REFERENCES}

[1] A. Richter, "Geschwindigkeitsvorgabe an Lichtsignalanlagen," Ph.D. dissertation, Helmut-Schmidt-Universität, Jan. 2005.

[2] M. Buehler, K. Iagnemma, and S. Singh, Eds., The DARPA Urban Challenge, ser. Springer Tracts in Advanced Robotics. Springer, 2009.

[3] B. D. Schutter, "Optimal traffic light control for a single intersection," in American Control Conference, vol. 3, 1999, pp. 2195-2199.
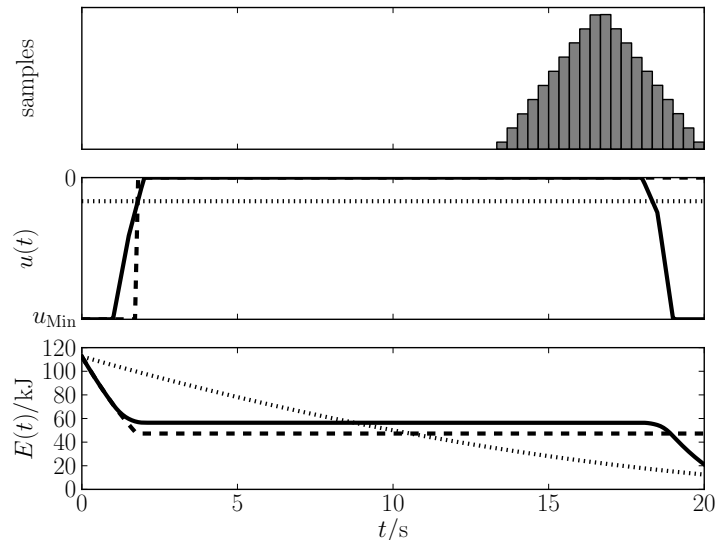

(a) Similar to Fig. 6a the optimal input trajectory is compared using samples from a triangular distribution. The top plot shows the histogram of samples, below are the input and the energy trajectories.

\begin{tabular}{lccc}
\hline Trajectory & optimal & CA & $\begin{array}{c}\text { opt. for } \\
T=T_{\text {Max }}\end{array}$ \\
\hline avg. energy loss/kJ & 57.4 & 90.1 & 65.3 \\
\hline avg. saving & - & $36.31 \%$ & $12.11 \%$ \\
\hline
\end{tabular}

(b) As before the optimal input trajectory outperforms the other candidate trajectories. The results have been achieved by samples of a triangular distribution function.

Fig. 7. Comparison of the obtained optimal trajectory for a triangular probability distribution function.

[4] K. Katsaros, R. Kernchen, M. Dianati, and D. Rieck, "Performance study of a Green Light Optimized Speed Advisory (GLOSA) application using an integrated cooperative ITS simulation platform," in Wireless Communications and Mobile Computing Conference, July 2011, pp. 918-923.

[5] M. Sánchez, J.-C. Cano, and D. Kim, "Predicting traffic lights to improve urban traffic fuel consumption," in ITS Telecommunications Proceedings, International Conference on, June 2006, pp. 331-336.

[6] T. Tielert, M. Killat, H. Hartenstein, R. Luz, S. Hausberger, and T. Benz, "The impact of traffic-light-to-vehicle communication on fuel consumption and emissions," in Internet of Things, Dec. 2010, pp. 1-8.

[7] B. Asadi and A. Vahidi, "Predictive cruise control: Utilizing upcoming traffic signal information for improving fuel economy and reducing trip time," Control Systems Technology, IEEE Transactions on, vol. 19, no. 3, pp. 707-714, May 2011.

[8] E. Koukoumidis, L.-S. Peh, and M. Martonosi, "SignalGuru: Leveraging mobile phones for collaborative traffic signal schedule advisory," in 9th ACM International Conference on Mobile Systems, Applications and Services (MobiSys), June 2011.

[9] M. Kerper, C. Wewetzer, A. Sasse, and M. Mauve, "Learning traffic light phase schedules from velocity profiles in the cloud," in New Technologies, Mobility and Security (NTMS), May 2012, pp. 1-5.

[10] I. Iglesias, L. Isasi, M. Larburu, V. Martinez, and B. Molinete, "I2V communication driving assistance system: On-board traffic light assistant," in Vehicular Technology Conference, Sept. 2008, pp. 1-5.

[11] M. Krause and K. Bengler, "Traffic Light Assistant - Driven in a simulator," in Intelligent Vehicles Symposium Workshops, 2012.

[12] M. Vašak, M. Baotić, M. Bago, and N. Perić, "Constrained energy optimal control of trains: Conceptual implementation," in Transportation Systems with International Participation, Automation in Transportation, 2010, pp. 143-146.

[13] D. E. Kirk, Optimal Control Theory: An Introduction. Englewood Cliffs, Prentice-Hall, 1970.

[14] G. Mahler and A. Vahidi, "Reducing idling at red lights based on probabilistic prediction of traffic signal timing," in American Control Conference, 2012, pp. 6557-6562.

[15] L. S. Pontryagin, V. G. Boltyansky, R. V. Gamkrelidze, and E. F. Mischenko, The Mathematical Theory of Optimal Processes. Wiley/Interscience, 1962. 